\title{
Neonatal hippocampal damage in rats and risk of schizophrenia
} Georgios Papazisis*1,3, Aimilios Lallas ${ }^{2}$, Chrysa Pourzitaki ${ }^{1}$ and Dimitrios Kouvelas ${ }^{1}$

Address: ${ }^{1}$ Department of Pharmacology, School of Medicine, Aristotle University of Thessaloniki, Thessaloniki, Greece, ${ }^{2}$ Department of Histology, School of Medicine, Aristotle University of Thessaloniki, Thessaloniki, Greece and ${ }^{3}$ Psychiatric Hospital of Thessaloniki, Thessaloniki, Greece

* Corresponding author

from International Society on Brain and Behaviour: 3rd International Congress on Brain and Behaviour

Thessaloniki, Greece. 28 November - 2 December 2007

Published: 17 April 2008

Annals of General Psychiatry 2008, 7(Suppl I):S229 doi:I0.II86/I744-859X-7-SI-S229

This abstract is available from: http://www.annals-general-psychiatry.com/content/7/SI/S229

(C) 2008 Papazisis et al.; licensee BioMed Central Ltd.

\section{Background}

While the literature on schizophrenia is well developed, there is epidemiological evidence that obstetric complications, such as perinatal asphyxia, are significantly increased in schizophrenic patients compared to controls. Recent findings suggest an increased risk of developing social withdrawal, neophobia and behavioral stereotypies as a consequence of neonatal asphyxia in rats and various functional and structural changes in the hippocampus have been consistently implicated in human schizophrenia.

\section{Materials and methods}

PubMed and ISI Web of Science were searched to identify peer-reviewed studies published between 1987 and 2007 focusing on neonatal damage of the rat hippocampus as a potential model of schizophrenia.

\section{Results}

Neonatal insult of the hippocampus may disrupt development of the widespread cortical and subcortical circuitry in which the hippocampus participates. The lesions were intended to involve regions of the hippocampus that directly project to the prefrontal cortex, namely, the ventral hippocampus and ventral subiculum. Morphologic changes often assessed in animal models and deemed similar to pathological changes in human schizophrenic brain include ventricular enlargement and a variety of hippocampal and/or cerebral cortical alterations including reduced volume, neuronal atrophy and altered neurogenesis and neuronal migration.

\section{Conclusions}

Neonatal damage of the rat hippocampus appears to reproduce a broad spectrum of schizophrenia-related phenomena and establishes the neurobiologic plausibility of early damage having a delayed impact on neural functions implicated in schizophrenia. As such, long-term effects of perinatal asphyxia on brain development and the potential psychiatric complications have become an area of increasing research interest.

\section{References}

I. Boksa P: Animal models of obstetric complications in relation to schizophrenia. Brain Research Reviews 2004, 45(I): I- I 7.

2. Dalman C, Thomas HV, David AS, Gentz J, Lewis G, Allebeck P: Signs of asphyxia at birth and risk of schizophrenia. Populationbased case-control study. BrJ Psychiatry 200I, 1 79:403-408.

3. Lipska B: Using animal models to test a neurodevelopmental hypothesis of schizophrenia. Rev Psychiatr Neurosci 2004, 29(4):282-286.

4. Zornberg GL, Buka SL, Tsuang MT: Hypoxic-ischemia-related fetal/neonatal complications and risk of schizophrenia and other nonaffective psychoses: a 19-year longitudinal study. Am J Psychiatry 2000, I 57(2): 196-202. 